\title{
Prothrombotic and Proinflammatory Activities of the $\beta$-Hemolytic Group B Streptococcal Pigment
}

\author{
Nikolai Siemens ${ }^{a, b}$ Sonja Oehmcke-Hecht ${ }^{c}$ Jörn Hoßmann ${ }^{d}$ Sebastian B. Skorka ${ }^{b}$ \\ Roel H.T. Nijhuis ${ }^{e}$ f Corinne Ruppen ${ }^{g}$ Steinar Skrede ${ }^{\text {h, }}$ Manfred Rohde Daniel Schultz $^{\mathrm{k}}$

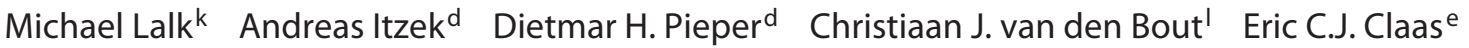 \\ Ed J. Kuijper ${ }^{\mathrm{e}}$ Robert Mauritz $^{\mathrm{m}}$ Parham Sendi ${ }^{9}$ Herman F. Wunderink $^{\mathrm{e}} \mathrm{I}$ Anna Norrby-Teglund ${ }^{\mathrm{a}}$ \\ ${ }^{a}$ Center for Infectious Medicine, Department of Medicine Huddinge, Karolinska Institutet, Karolinska University Hospital, \\ Huddinge, Sweden; ${ }^{b}$ Center for Functional Genomics of Microbes, Department of Molecular Genetics and Infection \\ Biology, University of Greifswald, Greifswald, Germany; ' Institute of Medical Microbiology, Virology, and Hygiene, \\ University Medicine Rostock, Rostock, Germany; ${ }^{\mathrm{d}}$ Microbial Interactions and Processes, Helmholtz Centre for Infection \\ Research - HZI, Braunschweig, Germany; ${ }^{e}$ Department of Medical Microbiology, Leiden University Medical Center, \\ Leiden, The Netherlands; ${ }^{f}$ Department of Medical Microbiology and Medical Immunology, Meander Medical Center, \\ Amersfoort, The Netherlands; ${ }^{9}$ Institute for Infectious Diseases, University of Bern, Bern, Switzerland; ${ }^{\mathrm{h}}$ Department \\ of Medicine, Haukeland University Hospital, Bergen, Norway; 'Department of Clinical Science, University of Bergen, \\ Bergen, Norway; ${ }^{j}$ Central Facility for Microscopy, Helmholtz Centre for Infection Research - HZI, Braunschweig, Germany; \\ kInstitute of Biochemistry, University of Greifswald, Greifswald, Germany; 'Department of Medical Microbiology, \\ University Medical Center Utrecht, Utrecht, The Netherlands; ${ }^{m}$ Department of Intensive Care Medicine, Leiden University \\ Medical Center, Leiden, The Netherlands
}

\section{Keywords}

Streptococcus agalactiae - Group B streptococcus · Pigment • Hemolysis · Coagulation · Inflammation

\begin{abstract}
A prominent feature of severe streptococcal infections is the profound inflammatory response that contributes to systemic toxicity. In sepsis the dysregulated host response involves both immunological and nonimmunological pathways. Here, we report a fatal case of an immunocompetent healthy female presenting with toxic shock and purpura fulminans caused by group B streptococcus (GBS; serotype III, CC19). The strain (LUMC16) was pigmented and hyperhemolytic. Stimulation of human primary cells with hyperhemolytic
\end{abstract}

karger@karger.com
www.karger.com/jin

Karger"

\section{(C) 2019 The Author(s)}

Published by S. Karger AG, Basel

Karge

Open access

This article is licensed under the Creative Commons AttributionNonCommercial-NoDerivatives 4.0 International License (CC BYNC-ND) (http://www.karger.com/Services/OpenAccessLicense) Usage and distribution for commercial purposes as well as any distribution of modified material requires written permission.
LUMC16 and STSS/NF-HH strains and pigment toxin resulted in a release of proinflammatory mediators, including tumor necrosis factor, interleukin (IL)-1 $\beta$, and IL-6. In addition, LUMC 16 induced blood clotting and showed factor XII activity on its surface, which was linked to the presence of the pigment. The expression of pigment was not linked to a mutation within the CovR/S region. In conclusion, our study shows that the hemolytic lipid toxin contributes to the ability of GBS to cause systemic hyperinflammation and interferes with the coagulation system.

(c) 2019 The Author(s)

Published by S. Karger AG, Basel
Dr. Nikolai Siemens

Department of Molecular Genetics and Infection Biology University of Greifswald

Felix-Hausdorff-Strasse 8, DE-17489 Greifswald (Germany)

E-Mail nikolai.siemens@uni-greifswald.de

Prof. Anna Norrby-Teglund

Center for Infectious Medicine, Department of Medicine Huddinge

Karolinska Institutet, Karolinska University Hospital

SE-14152 Huddinge (Sweden)

E-Mail anna.norrby-teglund@ki.se 


\section{Introduction}

Streptococcal toxic shock syndrome (STSS) is typically caused by Streptococcus pyogenes (group A streptococcus [GAS]). Reports of septic shock and multiorgan dysfunction due to Streptococcus agalactiae (group B streptococcus [GBS]) are increasing [1,2].STSS is characterized by a systemic cytokine storm, and key streptococcal triggers are the superantigens (SAgs) [3]. SAgs belong to a family of exotoxins, which activate $\mathrm{T}$ cells by binding intact to the MHC (major histocompatibility complex) class-II molecules on antigen-presenting cells and to the variable $\beta$-chains of the T-cell receptor. Once the MHC specificity of $\mathrm{T}$ cells is bypassed, excessive activation of $\mathrm{V} \beta$-specific $\mathrm{T}$ cells with subsequent downstream activation of other cell types occurs, which leads to an overzealous inflammatory response [3]. To date, SAg genes have been identified only in a few bacterial species, including group A, C, and G streptococci, Staphylococcus aureus, Yersinia pseudotuberculosis, and Mycoplasma arthritidis [4]. Despite the association with GBS and septic shock [5], GBS strains lack SAgs and the nature of the proinflammatory factors remains elusive.

Disseminated intravascular coagulation (DIC) is a frequent complication of systemic inflammation in severe invasive infections [6]. Blood coagulation can be initiated through extrinsic and intrinsic systems and ultimately results in the generation of thrombin $[7,8]$. Recent studies have suggested that the contact system, which represents the intrinsic coagulation pathway, plays a role in thrombus formation in DIC $[7,9]$. The human contact system consists of two main proteases, factor XII (FXII) and plasma prekallikrein, and one nonenzymatic cofactor, highmolecular-weight kininogen. Any foreign biological or artificial surfaces activate FXII, which in turn activates coagulation factor XI (FXI), resulting in subsequent thrombus formation [10]. There is a close relationship to DIC and systemic inflammatory responses. Several sepsis-associated cytokines, including tumor necrosis factor (TNF), interleukin (IL)-1 $\beta$, and IL-6, have been shown to stimulate procoagulant activities [11]. Blockage of IL-6, but not of TNF, attenuated endotoxin-induced activation of coagulation $[12,13]$. The role of IL-1 $\beta$ has not been fully elucidated yet. However, blocking of the IL-1 receptor in patients with sepsis resulted in reduced thrombin generation [14].

Classically, infections with Gram-negative bacteria have been associated with DIC [15]. However, the incidence of DIC in patients with Gram-positive bacterial infections seems to be similar [16]. GBS is a major pathogen in neonatal sepsis and in nonpregnant adults with underlying medical conditions and/or immunosuppressive treatment $[17,18]$. One of the key GBS virulence factors is the $\beta$-hemolytic pigment. The GBS pigment, whose transcription is regulated by the CovR/S two-component system [19], is hemolytic and cytolytic [20, 21], and its biosynthesis is catalyzed by the enzymes encoded within the $c y l$ operon [22]. Mutations in the CovR/S system or in the $c y l$ operon have been associated with expression of the pigment at the bacterial surface and with hyperhemolysis $[23,24]$, and such GBS strains are considered to be more pathogenic than nonhemolytic strains $[23,25]$.

Here, we report the fatal case of a previously healthy woman who died from septic shock accompanied by DIC and purpura fulminans, caused by a hyperhemolytic and pigmented GBS strain. A detailed characterization of the strain coupled to functional studies implicated the pigment as a key factor in eliciting prothrombotic and inflammatory responses.

\section{Subjects and Methods}

\section{Bacterial Strains}

The LUMC16 strain was identified from blood cultures by direct matrix-assisted laser desorption ionization time-of-flight mass spectrometry (Bruker Daltonik $\mathrm{GmbH}$ ). The isolate showed a $100 \%$ match with $S$. agalactiae by $16 \mathrm{~S}$ rRNA gene sequencing (GenBank accession No. KY321279) and expressed the group B Lancefield antigen. Susceptibility to antibiotics was determined by Etest (AB Biodisk), and the minimal inhibitory concentration values were: penicillin 0.047 , cefuroxime 0.047 , erythromycin 0.125 , clindamycin 0.064 , and meropenem $0.023 \mu \mathrm{g} \mathrm{mL} \mathrm{m}^{-1}$.

The serotyping of the isolates was performed by latex agglutination. For determination of the sequence type (ST)/clonal complex (CC), genomic DNA was extracted with the QIAamp DNA Mini Kit followed by multilocus sequence typing, as described previously [26], using the PubMLST database (https://pubmlst.org/ sagalactiae/). The case isolate was compared to two well-characterized phenotypical GBS variants, one hyperhemolytic (STSS/NF$\mathrm{HH}$ ) and one with low hemolytic activity (STSS/NF-LH) (serotype Ib, ST8), obtained from a patient with STSS and necrotizing fasciitis (NF) [23]; three noninvasive GBS isolates, i.e., Cervix (serotype III), Wound, and Urine (serotype Ib); and three isolates from necrotizing soft tissue infection (NSTI) cases (4012, 6051, and 6061) enrolled in the EU-funded project INFECT during 2013-2017 (ClinicalTrials.gov, NCT01790698). All GBS isolates were cultured in Todd Hewitt broth supplemented with $1.5 \%$ (w/v) yeast extract at $37^{\circ} \mathrm{C}$.

\section{In vitro Cell Assays}

Human neutrophils, peripheral blood mononuclear cells (PBMCs), and monocytes were isolated from whole blood or buffy coats from volunteers as previously detailed [27-29]. Neutrophil degranulation was determined by measurement of resistin and heparin-binding protein $(\mathrm{HBP})$ in neutrophil $\left(5 \times 10^{5}\right.$ cells $)$ super- 
natants collected after $2 \mathrm{~h}$ of bacterial (multiplicity of infection 10) or pigment stimulations [30-32]. Resistin and HBP levels were determined by ELISA (BioVendor and Axis-Shield) according to the manufacturer's instructions.

Human PBMCs $\left(5 \times 10^{5}\right.$ cells $)$ and monocytes $\left(5 \times 10^{5}\right.$ cells $)$ were stimulated with bacteria (multiplicity of infection 10) or pigment for $6 \mathrm{~h}$, and the levels of TNF, IL-1 $\beta$, IL-4, IL-5, IL-6, IL-8, and IL-10 were determined by DuoSet ELISA (all R\&D Systems) according to the manufacturer's instructions. For assessment of cytotoxicity, buffer stimulations and Triton X-100-lysed cells were used as negative and positive controls, respectively. Cytotoxicity was determined via CytoTox 96 Non-Radioactive Cytotoxicity Assay Kit (Promega) according to the manufacturer's guidelines.

\section{Pigment Purification and Hemoglobin Release Assay}

GBS pigment from LUMC16 and STSS/NF-HH was purified as previously described [20]. Briefly, bacterial cells were pelleted and washed three times with distilled water and twice with DMSO. The cell pellet was then suspended in DMSO-0.1\% TFA-0.1\% starch overnight to extract the pigment, cell debris was pelleted, and the supernatant containing the pigment was saved. The pigment was then precipitated by addition of $\mathrm{NH}_{4} \mathrm{OH}$ (final concentration, $0.25 \%)$. The precipitated pigment was washed three times with HPLC-grade water and twice with DMSO, dissolved in DMSO: $0.1 \%$ TFA, and purified using Sephadex LH-20 (GE Healthcare). Fractions containing purified pigment were pooled and precipitated with $\mathrm{NH}_{4} \mathrm{OH}$ (Sigma-Aldrich) as described above, and washed three times with HPLC-grade water (Sigma-Aldrich) and twice with DMSO.

Pigment from the STSS/NF-HH strain was used for all experiments due to a higher yield in extraction as compared to the LUMC16 strain. To confirm that the pigment isolated from STSS/ $\mathrm{NF}-\mathrm{HH}$ was the same substance as from the LUMC16 strain, MALDI-FTICR-MS was performed. Pigment extracts from LUMC16, STSS/NF-HH, and STSS/NF-LH (buffer control) were transferred to conductive ITO coated slides (Bruker) and lyophilized for $30 \mathrm{~min}$. The samples were coated with 2,5-dihydroxybenzoic acid as the matrix substance $(10 \mathrm{mg} / \mathrm{mL}$ dissolved in $90 \%$ acetonitrile, $10 \%[\mathrm{v} / \mathrm{v}]$ water, and $0.1 \%[\mathrm{v} / \mathrm{v}]$ trifluoroacetic acid) using an HTX TM-Sprayer under the following conditions: spray temperature $60^{\circ} \mathrm{C}$, flow rate $0.12 \mathrm{~mL} / \mathrm{min}$, drying time $10 \mathrm{~s}$, and 14 passages. Pigment measurements were done using a solariX FTMS system (Bruker) in positive mode. The parameters were: plate offset $100 \mathrm{~V}$, deflector plate $200 \mathrm{~V}$, laser shots 100 , and frequency $1,000 \mathrm{~Hz}$. The transfer optic parameters were: time of flight $0.9 \mathrm{~ms}$, frequency $4 \mathrm{MHz}$, and RF amplitude $350 \mathrm{Vpp}$. Pigment was exclusively present in samples from LUMC16 and STSS/NF-HH strains ( $\mathrm{m} / \mathrm{z}$ value 677.3862 ), whereas no peak was detected in extracts of the STSS/NF-LH strain (online suppl. Fig. 1; for all online suppl. material, see www.karger.com/doi/10.1159/000504002). This was used as a buffer control (negative control).

To confirm the hemolytic activity of the pigment, a hemoglobin release assay was performed using a method previously described with minor modifications [33]. In brief, $10 \%(\mathrm{v} / \mathrm{v})$ human blood in PBS was co-incubated with $1 \times 10^{5}$ bacteria or serial dilutions of the pigment in a final volume of $1 \mathrm{~mL}$ for $6 \mathrm{~h}$ or $1 \mathrm{~h}$ at $37^{\circ} \mathrm{C}$, respectively. Hemoglobin release was measured by recording the absorbance at $490 \mathrm{~nm}$. PBS- or water-treated blood served as a negative or positive control, respectively.

Group B Streptococcal Pigment

\section{Clotting and Chromogenic Substrate Assays}

Recalcification clotting times of the blood, activated partial thromboplastin time (aPTT), and prothrombin time (PT) and FXII activation at the bacterial surface were measured as previously described [34]. The experiments were repeated three times in triplicate.

\section{Whole Genome Sequencing and Processing}

DNA isolation, sequencing, and processing of the data are described in the online supplementary Methods. The sequencing data are deposited in the NCBI SRA and Genome database under accession No. SAMN10414105-13 and BioProject accession No. PRJNA505411 (ftp://ftp-trace.ncbi.nlm.nih.gov/sra/review/ SRP168694_20181211_101246_b1659515b9d1a59ebbc 790e01084a8f0). Designation of the strains within the BioProject database is summarized in online supplementary Table 1.

\section{Quantitative PCR Analysis}

Bacterial RNA was isolated using FastRNA Blue (MP Biomedicals). cDNA synthesis was performed using the SuperScript FirstStrand Synthesis System (Invitrogen). Quantitative PCR amplification was performed with the following primers: GBS_cylK_for ATTTATCTGGCGATCGGTTG, GBS_cylK_rev CCTTTGGCAAACCAATTAAATAAC, GBS_cylE_for GTCGTAGTGGACAGGCAATCAC, GBS_cylE_rev CGAAATGATCGACAATGCAG, GBS_gyrA_for CTTGGTGATGGGACGTTCAGG, GBS_gyrA_ rev GCTGAAGCAGCACGACGAAC [35], and SYBR GreenER Kit (Invitrogen) using an ABI Prism 7500 sequence detection system (Applied Biosystems). The levels of gyrA transcription were used for normalization.

\section{Statistics}

Statistical significance of differences for multiple comparisons was determined using the Kruskal-Wallis test with Dunnett's post test. Statistical significance of differences between two samples was calculated using Welch's $t$ test. Statistical analyses were performed using GraphPad Prism version 6. A $p$ value $<0.05$ was considered significant.

\section{Results}

\section{Case Report}

A previously healthy 55-year-old woman was admitted to the intensive care unit of Leiden University Medical Center (LUMC) with septic shock. The day before she had woken up in good condition, but during the course of the day she had felt slight abdominal discomfort. Within the following hours, she had developed diarrhea, nausea, vomiting, and severe pain in her extremities and back. A prehospital primary survey revealed hypoxemia and hypotension and she was transferred to a hospital.

In the emergency room, ventilator support and aggressive fluid resuscitation were administered, and treatment with cefuroxime, ciprofloxacin, metronidazole, and clindamycin was started immediately. Laboratory tests 


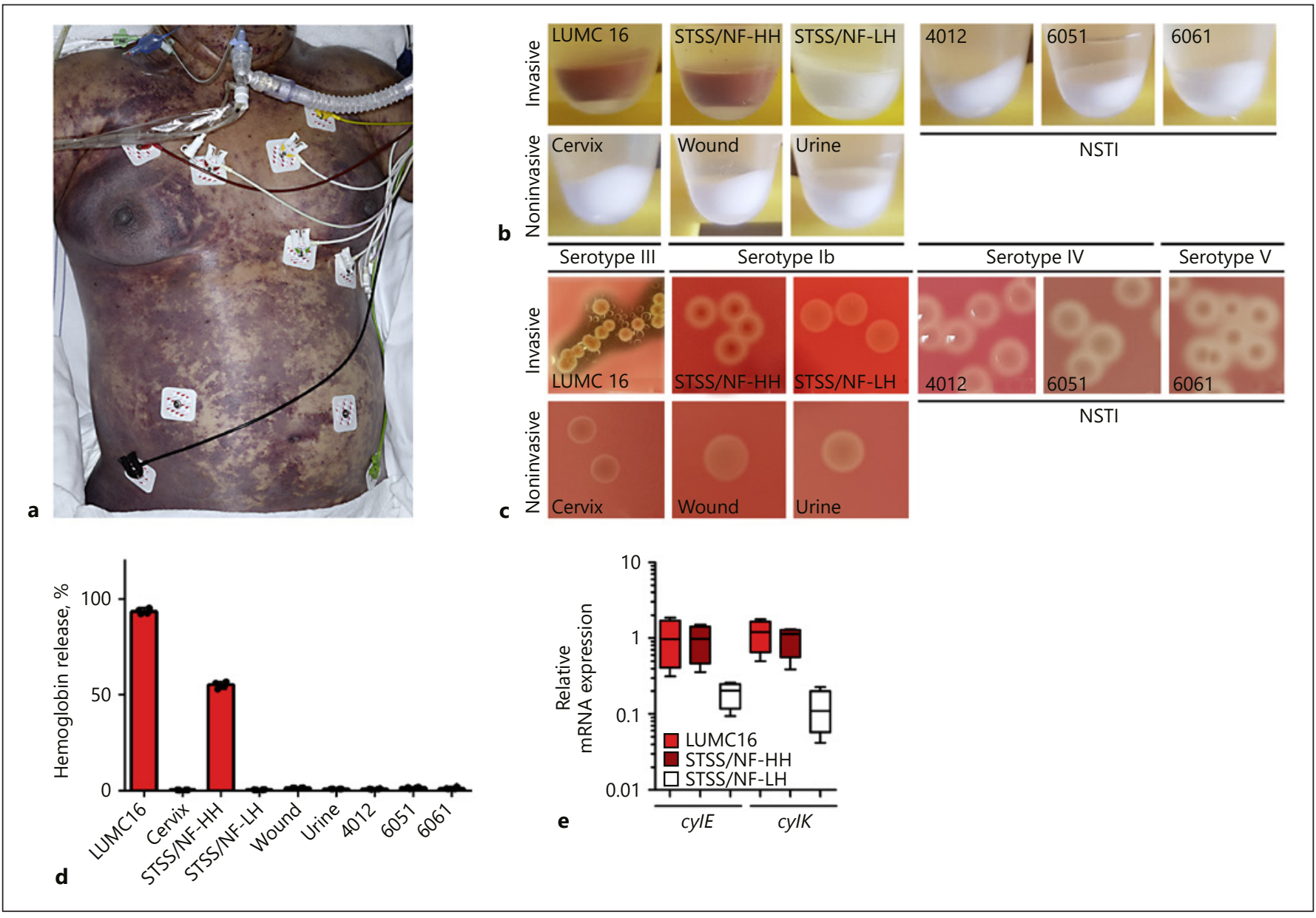

Fig. 1. Pigmented LUMC16 and STSS/NF-HH GBS strains cause hyperhemolysis. a Patient diagnosed with STSS, DIC, and purpura fulminans. b Pigmentation of the indicated strains after 16-h growth in THY medium. c, d Hemolytic activity of the indicated GBS strains shown by a clearance zone around the colonies on sheep blood agar plates (c) and by hemoglobin release assay with human blood (d). Each symbol represents one independent ex-

revealed lactic metabolic acidosis, acute kidney injury, elevated liver enzymes, thrombocytopenia, and leukocytosis. The coagulation parameters were compatible with DIC. A CT scan showed signs of enteritis and less enhancement of the pancreas, duodenum, and proximal jejunum without any abnormality of the mesenteric arteries. Upon arrival at the intensive care unit of the tertiary center, she had progressed to septic shock with purpura fulminans and DIC (Fig. 1a). Later, antibiotics were switched to benzylpenicillin and clindamycin, and intravenous polyspecific immunoglobulins were given, since blood cultures yielded Gram-positive cocci in chains, which were later identified as GBS. Within $36 \mathrm{~h}$ after the periment. Bars denote median values $(n=4)$. e Relative mRNA expression of the indicated genes encoding for pigment biosynthesis from stationary-phase bacterial cultures $(n=4)$. DIC, disseminated intravascular coagulation; GBS, group B streptococcus; $\mathrm{HH}$, hyperhemolytic; LH, low hemolytic; NF, necrotizing fasciitis; NSTI, necrotizing soft tissue infection; STSS, streptococcal toxic shock syndrome.

first symptom, the patient died from septic shock and multiorgan failure. The autopsy showed no macroscopic abnormalities - in particular, no asplenia or malignancies. No portal of entry could be identified.

\section{Pigmented GBS Strains Are Hyperhemolytic}

Cultures of the case's LUMC16 strain displayed a hyperhemolytic and pigmented phenotype (Fig. 1b; LUMC16), and the isolate belonged to serotype III and CC19 (ST-19-like). The unusual presentation, with DIC and septic shock in an immunocompetent individual, led us to explore strain properties that could explain the severe course of infection. For this purpose, we included 


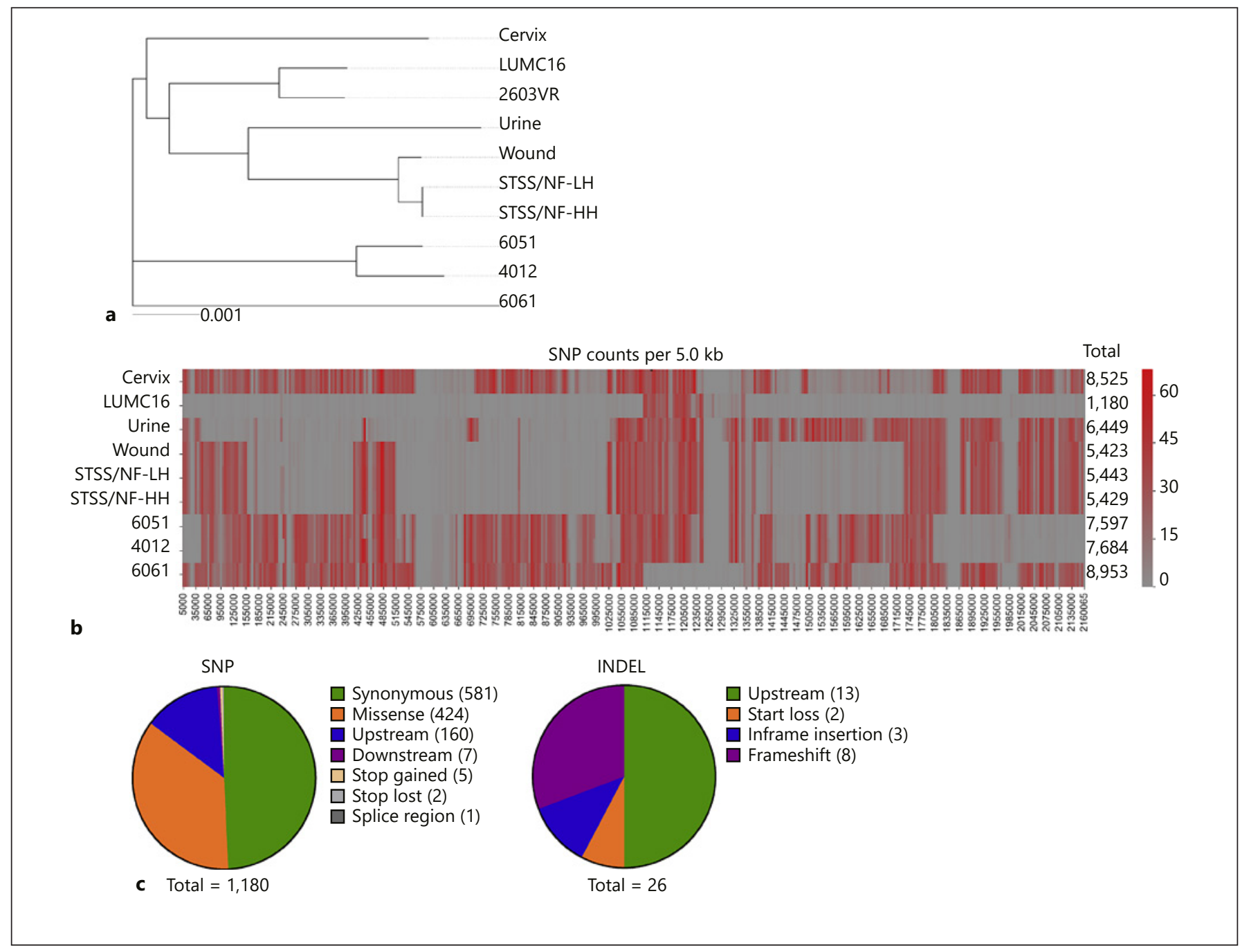

Fig. 2. Whole genome sequencing analyses. a Neighborhood joining tree calculated via genome-genome distance calculator and inferred from Streptococcus agalactiae assembled genomes. b Base differences per $5.0 \mathrm{~kb}$ based on the comparison of the whole genomes of the indicated strains with the annotated genome of the 2603VR strain. c Single nucleotide polymorphism (SNP; left panel) and insertion/deletion (INDEL; right panel) variants found within the whole genome of the case's LUMC16 GBS strain. GBS, group B streptococcus; HH, hyperhemolytic; LH, low hemolytic; NF, necrotizing fasciitis; STSS, streptococcal toxic shock syndrome.

strains of different serotypes isolated from invasive (STSS, NF, and NSTI) and noninvasive infections (Fig. 1b). The strain selection included two previously characterized phenotypic variants, $\mathrm{HH}$ and $\mathrm{LH}$, isolated from the same tissue culture of an STSS/NF case [23]. Among the strain collection, only LUMC16 and STSS/NF-HH were pigmented (Fig. 1b). LUMC16 displayed a remarkably strong hemolysis, even exceeding the activity of STSS/NF-HH, as evident by hemolysis zones on blood agar plates (Fig. 1c) and in the hemoglobin release assay (Fig. 1d). In addition, the pigmented strains LUMC16 and STSS/NF-
$\mathrm{HH}$ showed a greater abundance of $c y l E$ and $c y l K$ transcripts than a natural nonpigmented STSS/NF-LH GBS variant (Fig. 1e). However, no differences in gene expression levels between the two pigmented strains were noted.

\section{Comparative Whole Genome Analyses of the Strains}

In our previous report, the presence of the pigment and hemolytic phenotype of STSS/NF-HH were attributed to a deletion of valine in CovR [23]. However, this result was based on targeted $\operatorname{cov} \mathrm{R} / \mathrm{S}$ single gene sequencing. Therefore, we conducted whole genome 


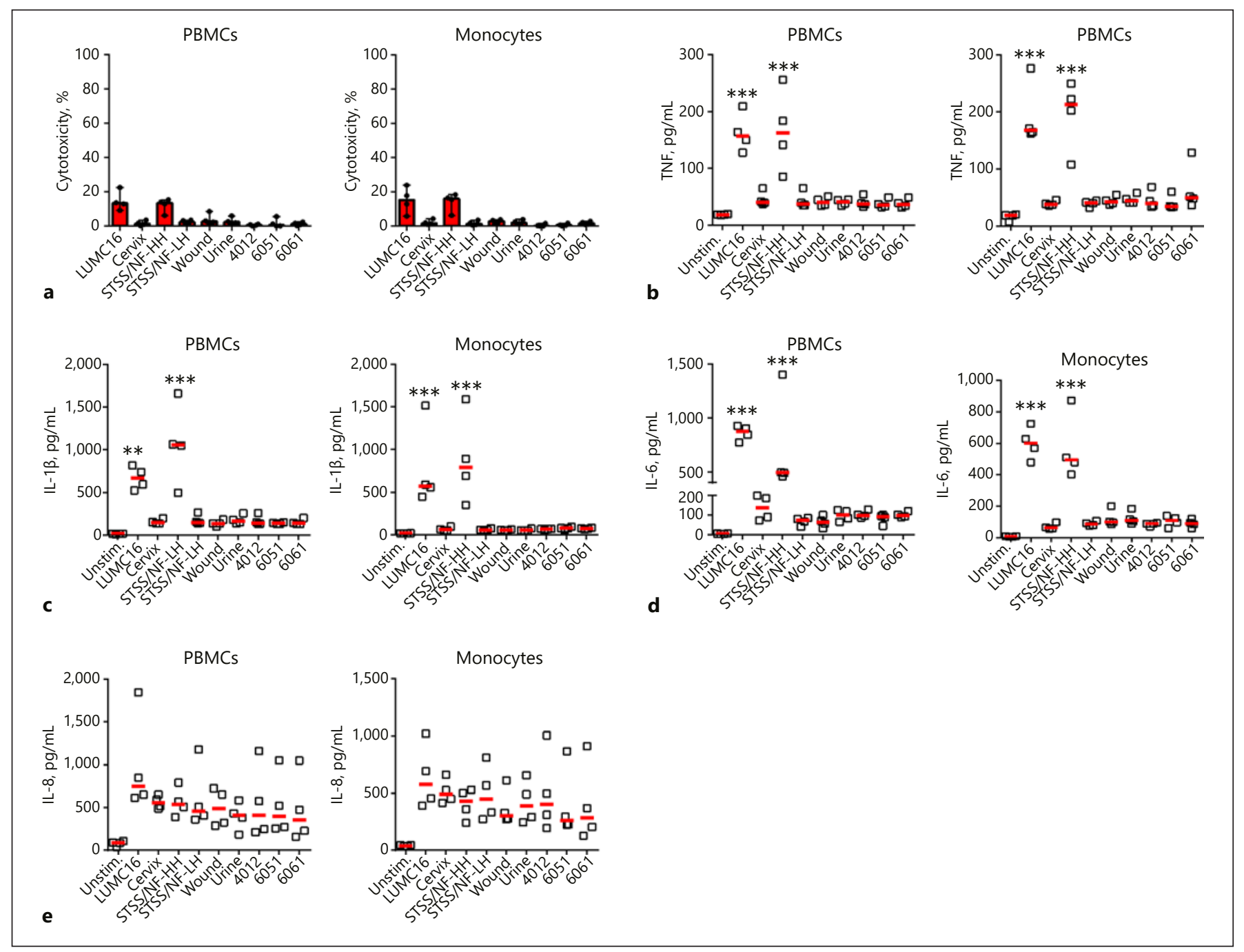

Fig. 3. Hyperhemolytic LUMC16 and STSS/NF-HH strains induce a toxic shock syndrome-associated proinflammatory response in human PBMCs and monocytes. a Human PBMCs (left panel) and monocytes (right panel) were stimulated with viable bacteria for $6 \mathrm{~h}$ and cytotoxic effects were assessed. Each symbol represents primary cells from one donor. Bars denote median values $(n=4)$. b-e TNF (b), IL-1 $\beta$ (c), IL-6 (d), and IL-8 (e) release by human primary PBMCs and/or monocytes in response to bacterial stimu-

analyses of all strains included in this study. A fully sequenced and annotated genome of the 2603VR GBS strain (serotype V), which was isolated from an invasive infection of a nonpregnant female, was used for the sequence variant (SV) analyses. In total, between 1.9 and 2.2 million bp and 1,911-2,147 CDS were identified (online suppl. Table 1). Between 83 and $87 \%$ of the 2603VR genes were shared in strains of this study (online suppl. Fig. 2). lation. Each symbol represents stimulation of PBMCs/monocytes from one healthy volunteer. Horizontal lines denote median values $(n=4)$. The level of significance was determined using the KruskalWallis test with Dunnett's multiple comparison $\left(* * p<0.01,{ }^{* * *} p<\right.$ 0.001). HH, hyperhemolytic; LH, low hemolytic; NF, necrotizing fasciitis; PBMCs, peripheral blood mononuclear cells; STSS, streptococcal toxic shock syndrome; Unstim., unstimulated.

A neighbor joining tree, created from assembled whole genomes, showed that LUMC16 is more closely related to the 2603VR reference strain than to all other strains (Fig. 2a). Comparative analyses of the nine strains with the 2603VR strain resulted in identification of a wide array of SVs, ranging from 1,180 SVs in the LUMC16 strain background to $8,953 \mathrm{SVs}$ in the 6061 NSTI strain (Fig. 2b). In all, $49.2 \%$ of the identified SVs in LUMC16 were of a synonymous nature (Fig. 2c; online suppl. Table 2). In addi- 
Fig. 4. Hemolytic and cytolytic activities of the pigment. a Hemolytic activity of the isolated pigment. Pigment was added to $10 \%$ human blood at the indicated dilutions. Equivalent amounts of sample buffer were used as controls (ctrl.). The data shown are the mean and range from 4 independent pigment preparations $(n=4)$. b Human PBMCs (left panel) and monocytes (right panel) were stimulated with different concentrations of the pigment for $6 \mathrm{~h}$, and cytotoxic effects were assessed. Each symbol represents primary cells from one donor. Bars denote median values $(n \geq 4)$. PBMCs, peripheral blood mononuclear cells.

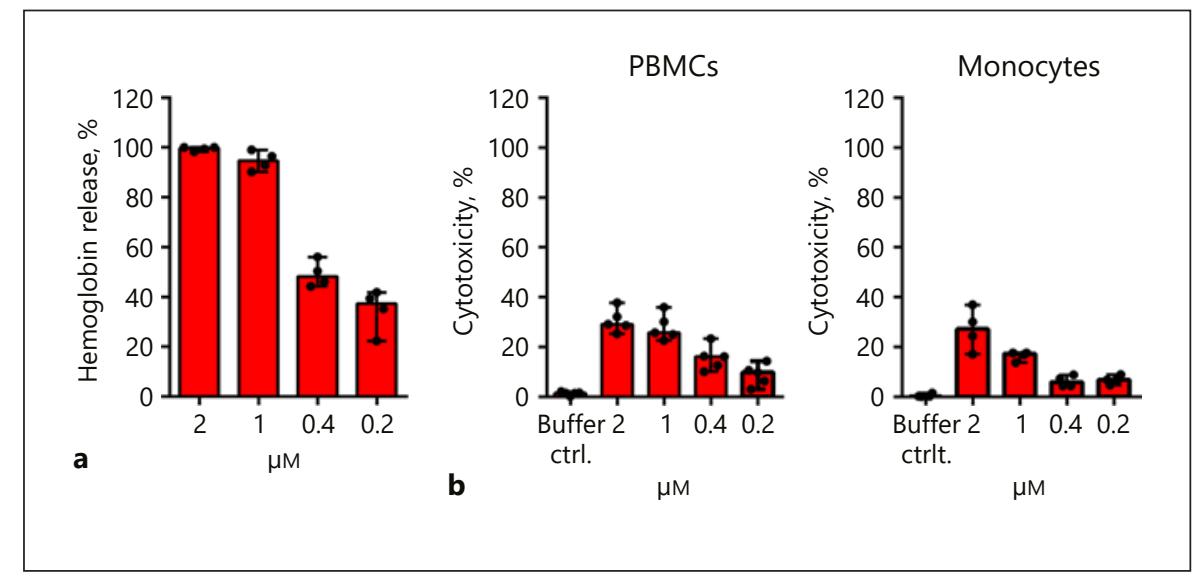

tion, a range of nonsynonymous SVs was identified. Furthermore, 26 insertions and deletions within the LUMC16 genome were detected (Fig. 2c; online suppl. Table 3). Analysis of the genome of the STSS/NF-HH strain confirmed a deletion of a triplet encoding for valine at position 30 within the $\operatorname{cov} R$ gene (online suppl. Table 4). Except for the GBS Urine strain harboring a V85I substitution in CovS, no other mutations in the entire CovR/S region and its surroundings were found (online suppl. Table 4).

Since both LUMC16 and STSS/NF-HH were pigmented, we also analyzed the entire $c y l$ operon, which encodes the genes for enzymes involved in pigment biosynthesis. There was only one SV identified, leading to a T186P substitution within CylJ (SAG0672), which was present in both LUMC16 and STSS/NF-HH (online suppl. Table 5). Furthermore, no mutations in CovR binding sites were found. Strain 4012 from an NSTI patient showed the highest frequency of SVs within the cyl operon as compared to the 2603VR strain.

\section{Pigmented and Hyperhemolytic GBS}

\section{Strains Induce Toxic Shock Syndrome-Associated}

Inflammatory Responses

We next examined the impact of all strains on different immune compartments. First, to exclude the potential secretion of molecules with proliferative activity by GBS strains, human PBMCs were stimulated with bacterial supernatants and proliferative responses were assessed. All strains failed to induce T-cell proliferation (online suppl. Fig. 3). Next, PBMCs, monocytes, and neutrophils were infected with viable bacteria, and pro- and anti-inflammatory responses, including degranulation by neutrophils, were assessed.

Both pigmented strains, LUMC16 and STSS/NF$\mathrm{HH}$, showed slightly higher cytotoxicity, as assessed by $\mathrm{LDH}$ release, towards PBMCs and monocytes (Fig. $3 a)$ - as well as significantly higher levels of the proinflammatory cytokines TNF, IL- $1 \beta$, and IL- 6 - than other strains (Fig. 3b-d). IL-8, as a general marker of inflammation, was equally induced in all stimulations (Fig. 2e), whereas only negligible levels of anti-inflammatory cytokines IL-4, IL-5, and IL-10 were detected (online suppl. Fig. 4). Assessment of neutrophil responses to different strains showed only low levels of cytotoxicity (online suppl. Fig. 5A). In contrast, analysis of resistin and HBP release revealed that all nine strains were potent inducers of neutrophil activation and degranulation (online suppl. Fig. 5B, C). However, no differences between pigmented and nonpigmented strains were found.

The GBS Pigment Induces Proinflammatory Responses

Since LUMC16 and STSS/NF-HH were pigmented, we next assessed the role of the pigment in inducing inflammation. Therefore, pigment was extracted (online suppl. Fig. 1) and its hemolytic activity was confirmed by the hemoglobin release assay (Fig. 4a). Next, PBMCs, monocytes, and primary neutrophils were stimulated with the pigment for $6 \mathrm{~h}$ or $2 \mathrm{~h}$, respectively, and effector molecule levels were determined. Concentration-dependent cytotoxic effects on PBMCs, monocytes (Fig. 4b), and neutrophils (online suppl. Fig. 7A) were observed. IL- $1 \beta$ (Fig. 5b) and IL-8 (Fig. $5 d$ ) release by PBMCs and monocytes, as well as neutrophil degranulation (online suppl. Fig. 7), remained independent of pigment concentrations, whereas TNF (Fig. 5a) and IL-6 (Fig. 5c) production by PBMCs or monocytes showed a dose-dependent response. No anti-inflammatory responses were induced in any of the pigment stimulations (online suppl. Fig. 6). 


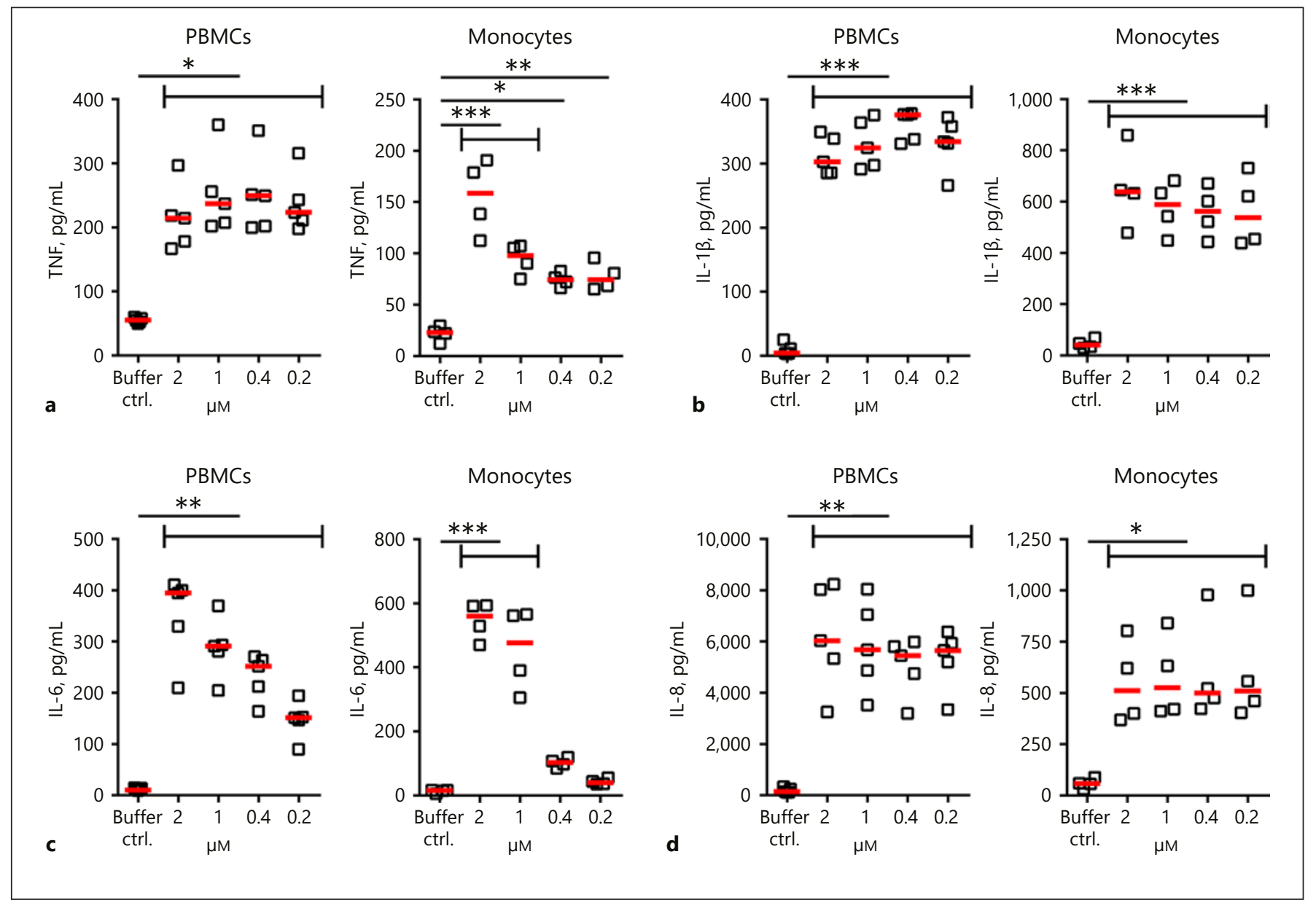

Fig. 5. Pigment-induced cytokine release by human PBMCs and monocytes. TNF (a), IL-1 $\beta$ (b), IL-6 (c), and IL-8 (d) release by human primary PBMCs and/or monocytes in response to pigment stimulation. Each symbol represents stimulation of PBMCs/monocytes from one healthy volunteer. Horizontal lines denote median values $(n \geq 4)$. The level of significance was determined using the Kruskal-Wallis test with Dunnett's multiple comparison $\left({ }^{*} p<0.05,{ }^{* *} p<0.01,{ }^{* * *} p<0.001\right)$. PBMCs, peripheral blood mononuclear cells.

\section{LUMC16 Strain Activates Coagulation}

To assess a potential role of the bacteria in interfering with the coagulation pathway, GBS strains and bacterial supernatants were incubated with human plasma for 30 min and then removed by centrifugation. aPTT (Fig. 6a) as well as PT (Fig. 6b) were determined in plasma. Significantly prolonged PT but not aPTT values (Fig. 6a, b) were observed for the majority of GBS strains as compared to plasma samples incubated with buffer alone. The majority of supernatants did not change aPTT, but prolonged PT (online suppl. Fig. 8). These results suggest that some GBS strains bind factors involved in the extrinsic pathway of coagulation on their surface.

To examine whether GBS could induce clotting of human blood, GBS strains were incubated with citrated hu- man blood, and recalcification clotting times were determined over a 4 -h period. All GBS strains were able to trigger coagulation (Fig. 6c; online suppl. Fig. 8C). Incubation of bacteria with human plasma did not induce clotting after recalcification, suggesting that induction of blood clotting by GBS is mainly dependent on human cellular components. As certain pathogenic bacteria are able to activate the human contact system [10], bacteria were incubated with human plasma, and activation of contact system proteases at the bacterial surface was assessed. Only LUMC16 showed FXIIa/PK activity at the bacterial surface (Fig. 6d). Although LUMC16 and STSS/NF-HH are both pigmented, only LUMC16 showed FXIIa/PK activity, suggesting a role of surface molecules or the composition of the bacterial cell membrane being responsible for binding contact 

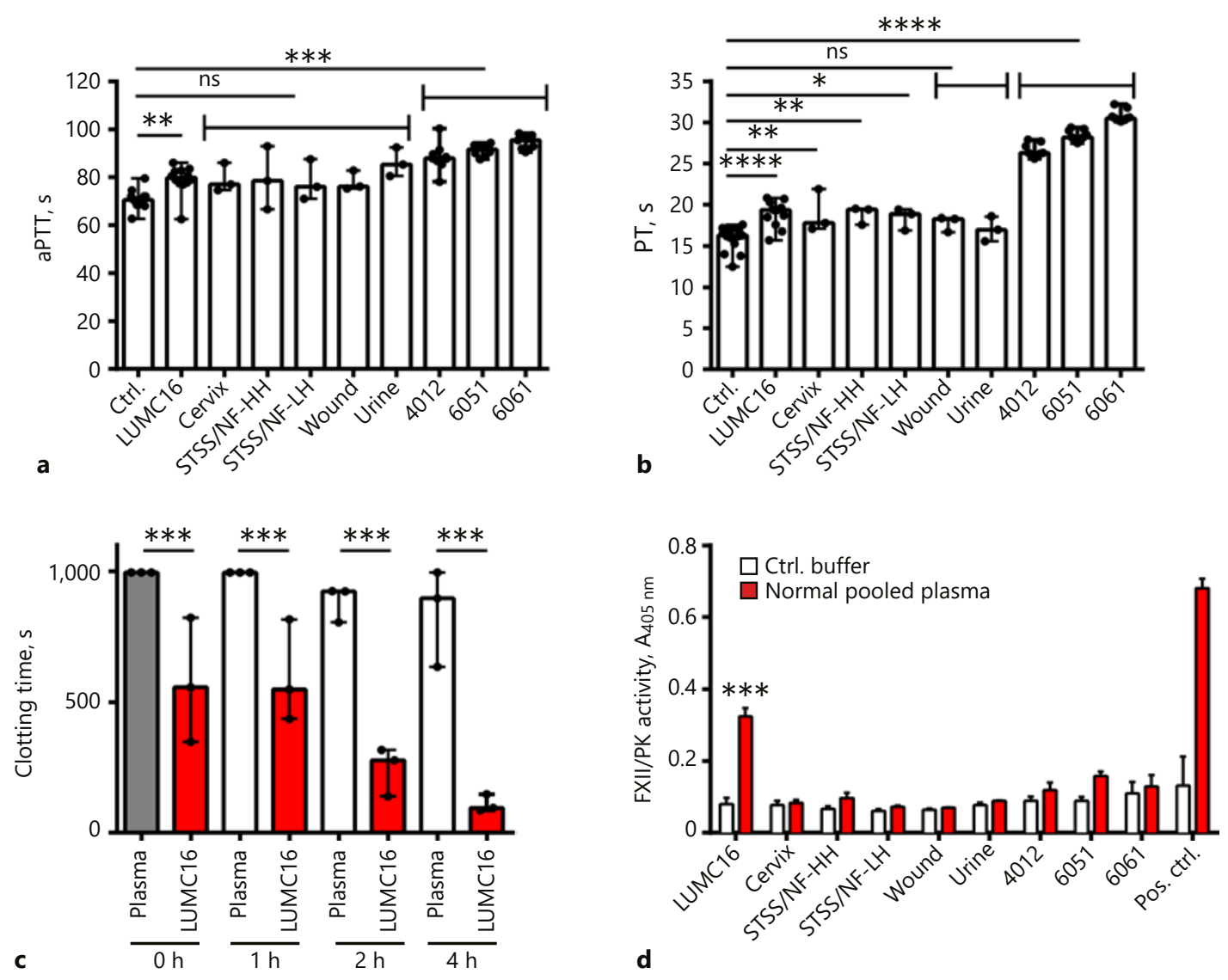

Fig. 6. Interference of GBS strains with the coagulation system. Bacteria were incubated in human plasma for $30 \mathrm{~min}$ at $37^{\circ} \mathrm{C}$ and removed via centrifugation. aPTT (a) and PT (b) of the resulting supernatants were determined in a coagulometer. Plasma incubated with buffer alone served as a control (Ctrl.). Each symbol represents one independent experiment. Bars denote median values and range from 3 and more independent experiments $(n \geq 3)$. The level of significance was determined using the Kruskal-Wallis test with Dunnett's multiple comparison ${ }^{*} p<0.05$, ${ }^{* *} p<0.01$, *** $\left.p<0.001,{ }^{* * * *} p<0.0001\right)$. c Blood clotting after incubation with the indicated GBS strains. Bacteria were added to the same volume of blood. Buffer alone served as a control. After incubation for 1,2 , and $4 \mathrm{~h}$ at $37^{\circ} \mathrm{C}$, the recalcification clotting times were measured. Each symbol represents one independent experiment. Bars denote median values and range from 3 independent experi-

ments $(n=3)$. The level of significance between the plasma controls and STSS strain samples was determined using Welch's $t$ test $\left.{ }^{* * *} p<0.001\right)$. d Activation of FXII/PK on the bacterial surface of the indicated GBS strains. Bacteria were incubated in HEPES buffer (control buffer [Pos. ctrl.]) or normal human plasma and washed, and chromogenic substrate S-2303 was added to the reaction. The FXII/PK activity was determined by measuring the absorbance (A) at $405 \mathrm{~nm}$. Data represent mean values \pm SD from 4 independent experiments $(n=4)$. Statistical significance between the controls and GBS strains was calculated using Welch's $t$ test $(* * * p<0.001)$. aPTT, activated partial thromboplastin time; FXII, factor XII; GBS, group B streptococcus; $\mathrm{HH}$, hyperhemolytic; LH, low hemolytic; NF, necrotizing fasciitis; PT, prothrombin time; STSS, streptococcal toxic shock syndrome.

factors. Examination of the bacterial surface via scanning electron microscopy showed no differences in surface structure between the strains (online suppl. Fig. 9).

\section{GBS Pigment Induces Plasma Clotting}

We next assessed the contribution of the GBS pigment to the clotting cascade. While the addition of dif- 


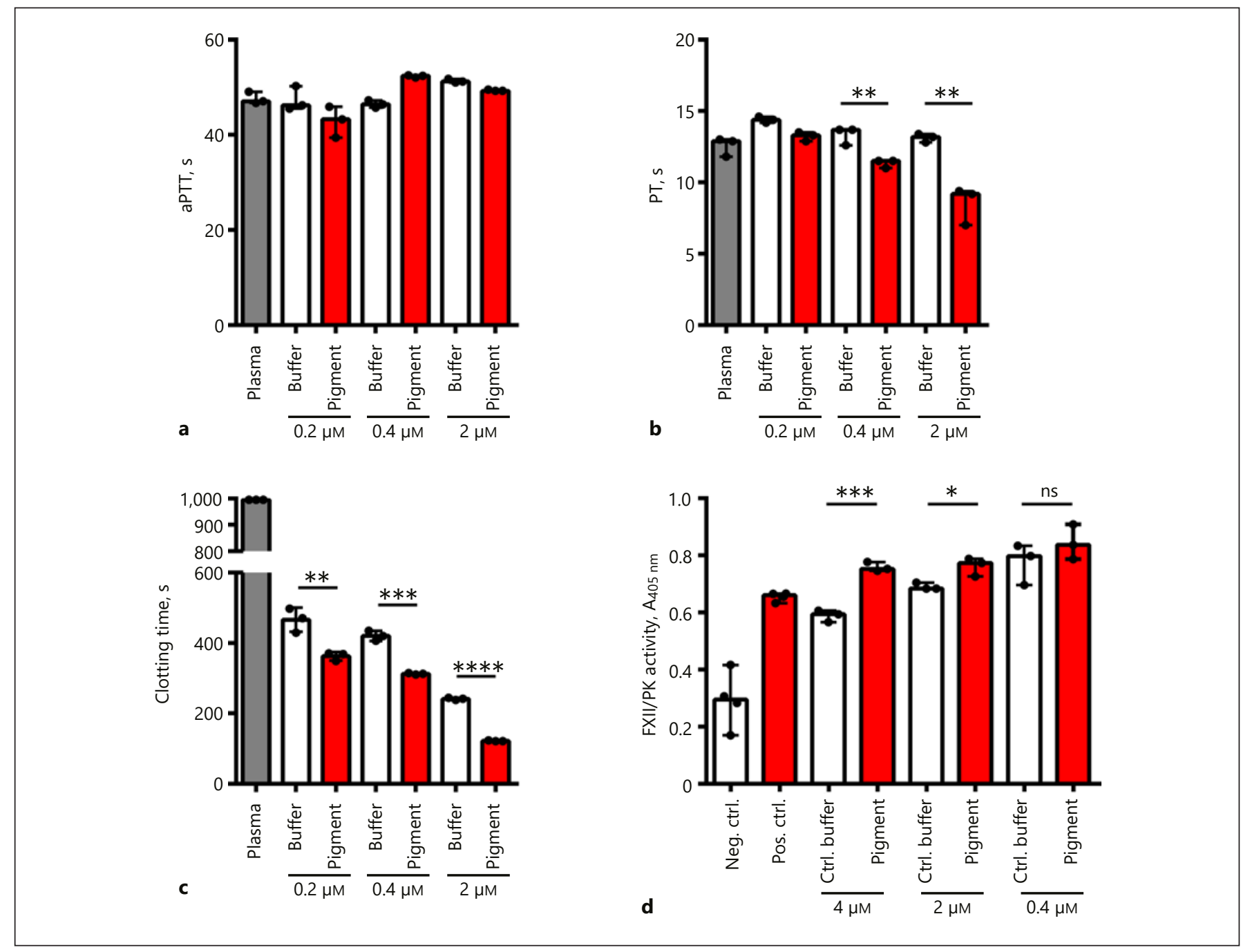

Fig. 7. Interference of the GBS pigment with the coagulation system. The indicated concentrations of the pigment were incubated in human plasma for $30 \mathrm{~min}$ at $37^{\circ} \mathrm{C}$ and removed via centrifugation. aPTT (a) and PT (b) of the resulting supernatants were determined in a coagulometer. Plasma incubated with the same amount of elution buffer of the pigment served as a control. Each symbol represents one independent experiment. Bars denote median values and range from 3 independent experiments $(n=3)$. The level of significance was determined using Welch's $t$ test $\left({ }^{* *} p<0.01\right)$. c Plasma clotting after incubation with the indicated dilutions of the GBS pigment. Elution buffer of the pigment served as a control. After incubation for $1 \mathrm{~h}$ at $37^{\circ} \mathrm{C}$, the recalcification clotting times were measured. Each symbol represents one inde-

volvement of the contact system, human plasma was incubated with the pigment and FXIIa/PK activity was determined. Samples preincubated with high amounts of pigment showed enhanced FXIIa/PK activity (Fig. 7d). pendent experiment. Bars denote median values and range from 3 independent experiments $(n=3)$. The level of significance between the controls and pigment samples was determined using Welch's $t$ test $\left(* * p<0.01,{ }^{* * *} p<0.001,{ }^{* * * *} p<0.0001\right)$. d Activation of FXII/PK GBS pigment. Pigment or elution buffer (ctrl. buffer) were incubated in normal human plasma and washed, and chromogenic substrate S-2303 was added to the reaction. The FXII/PK activity was determined by measuring the absorbance (A) at 405 $\mathrm{nm}$. Data represent mean values and range from 3 independent experiments $(n=3)$. Statistical significance between the controls and pigment was calculated using Welch's $t$ test $(* p<0.05$, $\left.{ }^{* * *} p<0.001\right)$. aPTT, activated partial thromboplastin time; FXII, factor XII; GBS, group B streptococcus; PT, prothrombin time.

\section{Discussion}

While STSS is typically caused by GAS, reports of septic shock due to GBS are increasing [1]. Invasive GBS disease is frequently observed in patients with comorbidities 
[36]. Here we report a fatal case with an unusually severe manifestation involving septic shock, DIC, and purpura fulminans in an immunocompetent individual. The causative GBS strain belonging to serotype III and CC19 was pigmented and hyperhemolytic. A series of experiments was undertaken to assess cytotoxic, inflammatory, and coagulation responses elicited by LUMC16 compared to other invasive and noninvasive GBS isolates. The results revealed for the first time that the hemolytic pigment induces proinflammatory and coagulatory activities in a dose-dependent manner.

The most severe condition caused by GBS is STSS [37]. SAgs have been identified as key mediators of TSS caused by GAS or S. aureus, mainly due to their induction of immune cell expansion and excessive cytokine release [3]. This phenomenon was not observed in our experiments with GBS. The hallmarks of the causative organism were its hyperhemolytic and proinflammatory activities. This is illustrated by a large zone of clearing around LUMC16 colonies grown on blood agar plates, an almost $100 \%$ hemoglobin release in the corresponding assay, and the stimulatory capacity of the immune cells. Pigment is a pore-forming agent, which has been associated with cytolytic injury to mast cells [38], with pyroptosis in human macrophages [21], with bacterial penetration of the human placenta [20], and with invasion of the amniotic cavity and fetal injury by circumventing neutrophils and neutrophil extracellular traps [39]. When extrapolating these findings to human disease, it is conceivable that hyperpigmented GBS may contribute to a fulminant clinical course as seen in our case and a previous report [23].

When human blood is exposed to bacterial surfaces, which can bind the zymogen FXII, this factor autoactivates into an enzyme, leading to subsequent thrombin formation in vitro [40]. Apart from bacteria, FXII itself can trigger inflammation by causing degranulation of human neutrophils [41] or inducing proinflammatory cytokine release from macrophages [42]. Our results suggest that, in contrast to other strains, LUMC16 is associated with a marked activation of the contact system, i.e., activated FXII and plasma kallikrein, on the bacterial surface by an as yet unidentified mechanism. The data further imply that the pigment itself is able to trigger the coagulation of plasma, independently of cellular blood components. The mechanisms underlying GBS pigment-mediated coagulation activation remain to be delineated.

The genes responsible for pigment synthesis are encoded in the cyl gene cluster and are under the control of the two-component system CovR/S [20]. In contrast to the previous report linking hyperhemolysis to a mutation in the $\operatorname{cov} \mathrm{R}$ gene [23], no mutations in $\operatorname{cov} \mathrm{R} / \mathrm{S}$ or the cyl operon were found in LUMC16. In general, LUMC16 was more similar to the $2603 \mathrm{VR}$ strain and showed a highly reduced number of SVs as compared to other strains. However, genes encoding for the pigment synthesis pathway were upregulated in the LUMC16 background to the same extent as in the STSS/NF-HH strain. This is in line with the report by Lupo et al. [35], who showed that although some hyperpigmented and hyperhemolytic GBS strains had covR mutations, others did not, implicating the involvement of other regulatory pathways. It may be noted that $\operatorname{cov} \mathrm{R} / \mathrm{S}$ mutations are overrepresented among GAS STSS strains [43], but a similar association has not been observed in GBS clinical isolates [44].

In conclusion, the present case demonstrates a fatal outcome of STSS accompanied by DIC caused by a GBS strain in an immunocompetent woman. The strain showed hyperhemolytic activity and proinflammatory and prothrombotic stimulation capacity, which were linked to the presence of the pigment and hyperhemolysis. These findings have direct implications on the contribution of the GBS pigment to systemic inflammation and interference with coagulation, and they warrant further experimental studies to identify mechanistic actions of the pigment and how these can be abolished.

\section{Acknowledgements}

We would like to thank the spouse of the patient for consenting to present the case. Jana Bull and Ina Schleicher are acknowledged for expert technical assistance. Katharina Riedel and Dirk Albrecht are acknowledged for assisting in MALDI-FTICR-MS.

\section{Statement of Ethics}

The husband of the case patient gave written informed consent for the publication of the report. Buffy coats or whole blood from consenting volunteers were obtained at the Karolinska University Hospital (Stockholm, Sweden) and University Medicine Greifswald (Greifswald, Germany). The regional ethical research committees in Stockholm and Greifswald approved the study (ethical approvals: 2006/229-31/3 [Stockholm]; BB 014/14 and BB 006/18 [Greifswald]). The study was conducted in accordance with the Helsinki Declaration.

\section{Disclosure Statement}

The authors have no conflicts of interest to declare. 


\section{Funding Sources}

This project was supported by the European Union (FP7/20072013) under the grant agreement 305340 (to A.N.-T.), Karolinska Institutet (to N.S. and A.N.-T.), the Stockholm County Council (ALF grant, to A.N.-T.), the Swedish Research Council (to A.N.T.), the Federal Excellence Initiative of Mecklenburg-Western Pomerania, the European Social Fund (ESF) Grant KoInfekt (ESF_14-BM-A55-0001_16), Stiftelsen Längmanska Kulturfonden (to N.S.), the Swedish Society of Medicine (SLS-501071 and SLS586271 to N.S.), the Deutsche Forschungsgemeinschaft (407176682 to N.S.), and the Velux Stiftung, Switzerland (724, to P.S.). The funding agencies had no part in manuscript compilation.

\section{Author Contributions}

N.S., S.O.-H., H.F.W., and A.N.-T. designed the study; N.S., S.O.-H., S.B.S., J.H., M.R., A.I., R.H.T.N., C.J.v.d.B., E.C.J.C., E.J.K., D.H.P., D.S., M.L., and R.M. performed experiments and analyzed data; R.H.T.N., E.C.J.C., E.J.K., C.R., P.S., R.M., H.F.W., and S.S. contributed to patient recruitment, sample collection, typing, and patient data analyses; N.S., S.O.-H., J.H., P.S., H.F.W., and A.N.-T. wrote the manuscript. All authors read, edited, and approved the final version.

\section{References}

1 Ikebe T, Tominaga K, Shima T, Okuno R, Kubota H, Ogata K, et al.; Working Group for Beta-Haemolytic Streptococci in Japan. Increased prevalence of group A streptococcus isolates in streptococcal toxic shock syndrome cases in Japan from 2010 to 2012. Epidemiol Infect. 2015 Mar;143(4):864-72.

2 Ikebe T, Chiba K, Shima T, Masuda C, Okuno $\mathrm{R}$, Ohya $\mathrm{H}$, et al.; Working Group for BetaHemolytic Streptococci in Japan. Evaluation of streptococcal toxic shock-like syndrome caused by group B streptococcus in adults in Japan between 2009 and 2013. J Infect Chemother. 2015 Mar;21(3):207-11.

3 Llewelyn M, Cohen J. Superantigens: microbial agents that corrupt immunity. Lancet Infect Dis. 2002 Mar;2(3):156-62.

4 Fraser JD, Proft T. The bacterial superantigen and superantigen-like proteins. Immunol Rev. 2008 Oct;225(1):226-43.

5 Chang B, Ikebe T, Wada A, Ogata K, Tomita M, Katsukawa C, et al.; Working Group for Streptococci in Japan. Surveillance of group B streptococcal toxic shock-like syndrome in nonpregnant adults and characterization of the strains in Japan. Jpn J Infect Dis. 2006 Jun; 59(3):182-5.

6 Gando S, Kameue T, Nanzaki S, Nakanishi Y. Disseminated intravascular coagulation is a frequent complication of systemic inflammatory response syndrome. Thromb Haemost. 1996 Feb;75(2):224-8.

7 Gailani D, Bane CE, Gruber A. Factor XI and contact activation as targets for antithrombotic therapy. J Thromb Haemost. 2015 Aug; 13(8):1383-95.

8 Levi M, Scully M. How I treat disseminated intravascular coagulation. Blood. 2018 Feb; 131(8):845-54

9 van Montfoort ML, Meijers JC. Recent insights into the role of the contact pathway in thrombo-inflammatory disorders. Hematology (Am Soc Hematol Educ Program). 2014 Dec;2014(1):60-5.

10 Oehmcke-Hecht S, Köhler J. Interaction of the human contact system with pathogens - an update. Front Immunol. 2018 Feb; 9:312.
11 Levi M, van der Poll T. Inflammation and coagulation. Crit Care Med. 2010 Feb;38(2 Suppl):S26-34.

12 van der Poll T, Levi M, Hack CE, ten Cate $\mathrm{H}$, van Deventer SJ, Eerenberg AJ, et al. Elimination of interleukin 6 attenuates coagulation activation in experimental endotoxemia in chimpanzees. J Exp Med. 1994 Apr;179(4): 1253-9.

13 van der Poll T, Levi M, van Deventer SJ, ten Cate H, Haagmans BL, Biemond BJ, et al. Differential effects of anti-tumor necrosis factor monoclonal antibodies on systemic inflammatory responses in experimental endotoxemia in chimpanzees. Blood. 1994 Jan;83(2): 446-51.

14 Boermeester MA, van Leeuwen PA, Coyle SM, Wolbink GJ, Hack CE, Lowry SF. Interleukin-1 blockade attenuates mediator release and dysregulation of the hemostatic mechanism during human sepsis. Arch Surg. 1995 Jul;130(7):739-48.

15 Lécuyer H, Borgel D, Nassif X, Coureuil M. Pathogenesis of meningococcal purpura fulminans. Pathog Dis. 2017 Apr;75(3):ftx027.

16 Kinasewitz GT, Yan SB, Basson B, Comp P, Russell JA, Cariou A, et al.; PROWESS Sepsis Study Group. Universal changes in biomarkers of coagulation and inflammation occur in patients with severe sepsis, regardless of causative micro-organism [ISRCTN74215569]. Crit Care. 2004 Apr;8(2):R82-90.

17 Batalis NI, Caplan MJ, Schandl CA. Acute deaths in nonpregnant adults due to invasive streptococcal infections. Am J Forensic Med Pathol. 2007 Mar;28(1):63-8.

18 Farley MM, Harvey RC, Stull T, Smith JD, Schuchat A, Wenger JD, et al. A populationbased assessment of invasive disease due to group B Streptococcus in nonpregnant adults. N Engl J Med. 1993 Jun;328(25): 1807-11.

19 Lamy MC, Zouine M, Fert J, Vergassola M, Couve E, Pellegrini E, et al. CovS/CovR of group B streptococcus: a two-component global regulatory system involved in virulence. Mol Microbiol. 2004 Dec;54(5):125068.
20 Whidbey C, Harrell MI, Burnside K, Ngo L, Becraft AK, Iyer LM, et al. A hemolytic pigment of Group B Streptococcus allows bacterial penetration of human placenta. J Exp Med. 2013 Jun;210(6):1265-81.

21 Whidbey C, Vornhagen J, Gendrin C, Boldenow E, Samson JM, Doering K, et al. A streptococcal lipid toxin induces membrane permeabilization and pyroptosis leading to fetal injury. EMBO Mol Med. 2015 Apr;7(4):488505

22 Spellerberg B, Martin S, Brandt C, Lütticken R. The cyl genes of Streptococcus agalactiae are involved in the production of pigment. FEMS Microbiol Lett. $2000 \mathrm{Jul} ; 188(2): 125-8$.

23 Sendi P, Johansson L, Dahesh S, Van Sorge NM, Darenberg J, Norgren M, et al. Bacterial phenotype variants in group B streptococcal toxic shock syndrome. Emerg Infect Dis. 2009 Feb;15(2):223-32.

24 Six A, Firon A, Plainvert C, Caplain C, Bouaboud A, Touak G, et al. Molecular characterization of nonhemolytic and nonpigmented group B streptococci responsible for human invasive infections. J Clin Microbiol. 2016 Jan;54(1):75-82

25 Liu GY, Doran KS, Lawrence T, Turkson N, Puliti M, Tissi L, et al. Sword and shield: linked group B streptococcal beta-hemolysin/ cytolysin and carotenoid pigment function to subvert host phagocyte defense. Proc Natl Acad Sci USA. 2004 Oct;101(40):14491-6.

26 Jones N, Bohnsack JF, Takahashi S, Oliver KA, Chan MS, Kunst F, et al. Multilocus sequence typing system for group B streptococcus. J Clin Microbiol. 2003 Jun;41(6): 2530-6.

27 Mairpady Shambat S, Siemens N, Monk IR, Mohan DB, Mukundan S, Krishnan KC, et al. A point mutation in AgrC determines cytotoxic or colonizing properties associated with phenotypic variants of ST22 MRSA strains. Sci Rep. 2016 Aug;6(1):31360.

28 Siemens N, Chakrakodi B, Shambat SM, Morgan M, Bergsten H, Hyldegaard O, et al.; INFECT Study Group. Biofilm in group A streptococcal necrotizing soft tissue infections. JCI Insight. 2016 Jul;1(10):e87882. 
29 Siemens N, Kittang BR, Chakrakodi B, Oppegaard O, Johansson L, Bruun T, et al.; INFECT Study Group. Increased cytotoxicity and streptolysin $\mathrm{O}$ activity in group $\mathrm{G}$ streptococcal strains causing invasive tissue infections. Sci Rep. 2015 Nov;5(1):16945.

30 Snäll J, Linnér A, Uhlmann J, Siemens N, Ibold $\mathrm{H}$, Janos $\mathrm{M}$, et al. Differential neutrophil responses to bacterial stimuli: streptococcal strains are potent inducers of heparin-binding protein and resistin-release. Sci Rep. 2016 Feb;6(1):21288.

31 Uhlmann J, Rohde M, Siemens N, Kreikemeyer B, Bergman P, Johansson L, et al. LL-37 triggers formation of Streptococcus pyogenes extracellular vesicle-like structures with immune stimulatory properties. J Innate Immun. 2016;8(3):243-57.

32 Uhlmann J, Siemens N, Kai-Larsen Y, Fiedler T, Bergman P, Johansson L, et al. Phosphoglycerate kinase - a novel streptococcal factor involved in neutrophil activation and degranulation. J Infect Dis. 2016 Dec;214(12):187683.

33 Nizet V, Gibson RL, Chi EY, Framson PE, Hulse M, Rubens CE. Group B streptococcal beta-hemolysin expression is associated with injury of lung epithelial cells. Infect Immun. 1996 Sep;64(9):3818-26.
34 Isenring J, Köhler J, Nakata M, Frank M, Jans C, Renault P, et al. Streptococcus gallolyticus subsp. gallolyticus endocarditis isolate interferes with coagulation and activates the contact system. Virulence. 2018 Jan;9(1):248-61.

35 Lupo A, Ruppen C, Hemphill A, Spellerberg $B$, Sendi P. Phenotypic and molecular characterization of hyperpigmented group B Streptococci. Int J Med Microbiol. 2014 Jul;304(56):717-24.

36 Skoff TH, Farley MM, Petit S, Craig AS, Schaffner W, Gershman K, et al. Increasing burden of invasive group B streptococcal disease in nonpregnant adults, 1990-2007. Clin Infect Dis. 2009 Jul;49(1):85-92.

37 Breiman RF, Davis JP, Facklam RR, Gray BM, Hoge CW, Kaplan EL, et al.; The Working Group on Severe Streptococcal Infections. Defining the group A streptococcal toxic shock syndrome. Rationale and consensus definition. JAMA. 1993 Jan;269(3):390-1

38 Gendrin C, Vornhagen J, Ngo L, Whidbey C, Boldenow E, Santana-Ufret V, et al. Mast cell degranulation by a hemolytic lipid toxin decreases GBS colonization and infection. Sci Adv. 2015 Jul;1(6):e1400225.

39 Boldenow E, Gendrin C, Ngo L, Bierle C, Vornhagen J, Coleman M, et al. Group B Streptococcus circumvents neutrophils and neutrophil extracellular traps during amniotic cavity invasion and preterm labor. Sci Immunol. 2016 Oct;1(4):1.
40 Schmaier AH. The contact activation and kallikrein/kinin systems: pathophysiologic and physiologic activities. J Thromb Haemost. 2016 Jan;14(1):28-39.

41 Wachtfogel YT, Kucich U, James HL, Scott CF, Schapira M, Zimmerman M, et al. Human plasma kallikrein releases neutrophil elastase during blood coagulation. J Clin Invest. 1983 Nov;72(5):1672-7.

42 Vorlova S, Koch M, Manthey HD, Cochain C, Busch M, Chaudhari SM, et al. Coagulation factor XII induces pro-inflammatory cytokine responses in macrophages and promotes atherosclerosis in mice. Thromb Haemost. 2017 Jan;117(1):176-87.

43 Ikebe T, Ato M, Matsumura T, Hasegawa $\mathrm{H}$, Sata T, Kobayashi K, et al. Highly frequent mutations in negative regulators of multiple virulence genes in group A streptococcal toxic shock syndrome isolates. PLoS Pathog. 2010 Apr;6(4):e1000832.

44 Sendi P, El Hay MA, Brandt CM, Spellerberg B. Group B streptococcal toxic shock syndrome and covR/S mutations revisited. Emerg Infect Dis. 2017 Jan;23(1):150-2. 